\title{
ON A HIGH-FIDELITY HIERARCHICAL APPROACH TO BUCKLING LOAD CALUCATIONS
}

\author{
Johann Arbocz* \\ Delft University of Technology, The Netherlands \\ and \\ James H. Starnes ${ }^{\star \star}$ and Michael P. Nemeth ${ }^{\star \star *}$ \\ NASA Langley Research Center, Hampton, VA 23681-001
}

\section{ABSTRACT}

As a step towards developing a new design philosophy, one that moves away from the traditional empirical approach used today in design towards a science-based design technology approach, a recent test series of 5 composite shells carried out by Waters [1] at NASA Langley Research Center is used. It is shown how the hierarchical approach to buckling load calculations proposed by Arbocz et al [2] can be used to perform an approach often called "high fidelity analysis", where the uncertainties involved in a design are simulated by refined and accurate numerical methods. The Delft Interactive Shell DEsign COde (short, DISDECO) is employed for this hierarchical analysis to provide an accurate prediction of the critical buckling load of the given shell structure. This value is used later as a reference to establish the accuracy of the Level-3 buckling load predictions. As a final step in the hierarchical analysis approach, the critical buckling load and the estimated imperfection sensitivity of the shell are verified by conducting an analysis using a sufficiently refined finite element model with one of the current generation two-dimensional shell analysis codes with the advanced capabilities needed to represent both geometric and material nonlinearities.

* Professor, Faculty of Aerospace Engineering, Associate Fellow AIAA

** Senior Engineer, Structures and Materials Competency, Fellow AIAA

*** Senior Research Engineer, Associate Fellow AIAA

Copyright $\odot 2001$ by J. Arbocz, J.H. Starnes and M.P. Nemeth

Published by AIAA with permission

\section{INTRODUCTION}

It is generally agreed that, in order to make the development of the Advanced Space Transportation System a success and to achieve the very ambitious performance goals (like every generation of vehicles $10 x$ safer and 10x cheaper than the previous one), one must make full and efficient use of the technical expertise accumulated in the past 50 years or so, and combine it with the tremendous computational power now available. It is obvious that with the strict weight constraints used in space applications these performance goals can only be achieved with an approach often called "high fidelity analysis", where the uncertainties involved in a design are simulated by refined and accurate numerical models. In the end the use of "high fidelity" numerical simulation will also lead to overall cost reduction, since the analysis and design phase will be completed faster and only the reliability of the final configuration needs to be verified by structural testing.

The light-weight shell structures used in aerospace applications are often buckling critical. The buckling load calculations are usually carried out by one of the many currently available finite element based computer codes [e.g., 3,4]. In order to reduce computer execution time, buckling analyses are often done using only the small displacement stiffness matrix $\mathrm{K}_{0}$. This approach is used, despite the fact that the "initial stability problem" so formulated can only give physically meaningful answers if the elastic solutions based on $K_{0}$ (at least approximately) are identically equal to zero [5].

When the qualitative nature of the expected behavior is completely unknown, the stability of the structure must be investigated using the full tangent stiffness matrix $\mathrm{K}_{T}$ in order to guarantee accurate and reliable buckling load and buckling mode predictions. In order to discover the load level at which $\mathrm{K}_{\mathrm{T}}$ ceases to be positive definite (that is, the load level when 
buckling occurs), a step-by-step analysis procedure is needed.

In addition, it is imperative (though often completely neglected), that at the beginning of any stability investigation, the accuracy of the discrete model used should be checked against available analytical or semi-analytical results. This step is part of a mandatory study needed in order to establish the dependence of the buckling load predictions on the mesh distribution used. Furthermore, as has been pointed out in the past by Byskov [6], if one carries out imperfection sensitivity investigations, which involve an extension of the solution into the postbuckling response region, further mesh refinement may be needed since the wavelength of the dominant large deformation pattern may often decrease significantly.

Finally, whenever one is engaged in shell stability analysis it is especially important that one is aware of the possible detrimental effects of a whole series of factors, that have been investigated extensively in the late 1960s and the early 1970s. Thus for an accurate and reliable prediction of the critical buckling load of a real structure, one must account not only for the influence of initial imperfections [e.g., $7,8]$ and of the boundary conditions [e.g. 9], but one must also consider the effects of stiffener and load eccentricity [e.g., 10] and the prebuckling deformations caused by the edge restraints [e.g. 11,12].

A recent test series of 5 composite shells carried out by Waters [1] at NASA Langley Research Center is used to illustrate how such a hierarchical approach to buckling load calculations can be carried out. The platform for the multi-level computations, needed for an accurate prediction of the critical buckling loads and a reliable estimation of their imperfection sensitivity, is provided by DISDECO [13]. With this open ended, hierarchical, interactive computer code the user can access from his workstation a succession of programs of increasing complexity.

\section{SOLUTION OF THE BUCKLING PROBLEM}

In the following it will be shown that with the help of DISDECO, the Delft Interactive Shell DEsign COde, the shell designer can study the buckling behavior of a specified shell, calculate its critical buckling load quite accurately and make a reliable prediction of the expected degree of imperfection sensitivity of the critical buckling load. The proposed procedure consists of a hierarchical approach, where the analyst proceeds step-by-step from the simpler
(Level-1) methods used by the early investigators to the more sophisticated analytical and numerical (Level-2 and Level-3) methods used presently.

\section{Level-1 Perfect Shell Buckling Analysis}

The geometric and material properties of the 8-ply, composite shell with symmetrical layup of Ref. [1] are listed in Table 1.

Table 1.

Geometric properties of NASA layered composite shell AW-CYL-1-1 [1] - $[ \pm 45 / 0 / 90]_{S}$

\begin{tabular}{|lllll|}
$t_{\text {total }}(=\mathrm{h})$ & $=0.039976$ & in $(=1.01539$ & $\mathrm{mm}$ & ) \\
$\mathrm{L}$ & $=14.0$ & in $(=355.600$ & $\mathrm{mm}$ & ) \\
$\mathrm{R}$ & $=7.99945$ & in $(=203.18603$ & $\mathrm{mm}$ & ) \\
$\mathrm{E}_{11}$ & $=18.5111 \times 10^{6}$ & $\mathrm{psi}\left(=12.7629 \times 10^{4}\right.$ & $\left.\mathrm{N} / \mathrm{mm}^{2}\right)$ \\
$\mathrm{E}_{22}$ & $=1.64 \times 10^{6}$ & $\mathrm{psi}\left(=1.13074 \times 10^{4}\right.$ & $\left.\mathrm{N} / \mathrm{mm}^{2}\right)$ \\
$\mathrm{G}_{12}$ & $=0.8706 \times 10^{6}$ & $\mathrm{psi}\left(=6.00257 \times 10^{3}\right.$ & $\left.\mathrm{N} / \mathrm{mm}^{2}\right)$ \\
$\mathrm{v}_{12}$ & $=0.300235$ & & \\
\hline
\end{tabular}

Note: Symmetrical lay-up with 8 plys of equal thicknesses $(=0.004997$ in)

Assuming a perfect shell $(\bar{W}=0)$ and the following membrane prebuckling state

$$
\begin{aligned}
& W^{(0)}=h W_{v}=h \bar{A}_{12}^{*} \frac{\lambda}{c} \\
& F^{(0)}=-\frac{E h^{2}}{c R} \frac{1}{2} \lambda y^{2}
\end{aligned}
$$

where

$$
\begin{aligned}
& \lambda=\frac{\sigma}{\sigma_{\mathrm{C} \ell}}=\frac{\mathrm{N}_{\mathrm{x}}}{\mathrm{N}_{\mathrm{c} \ell}} ; \sigma_{\mathrm{C} \ell}=\frac{\mathrm{Eh}}{\mathrm{ch}} ; \mathrm{N}_{\mathrm{C} \ell}=\sigma_{\mathrm{c} \ell \mathrm{h}} \\
& \text { and } \mathrm{c}=\sqrt{3\left(1-v^{2}\right)}
\end{aligned}
$$

then the nonlinear equations governing the prebuckling state are identically satisfied and the linearized stability equations reduce to a set of equations with constant coefficients. It has been shown in Ref. [14] that by assuming an asymmetric bifurcation mode of the form

$$
W^{(1)}=h \sin m \pi \frac{x}{L} \cos \frac{n}{R}\left(y-\tau_{K} x\right)
$$

where

$$
\begin{aligned}
& m=k=\text { number of axial half waves } \\
& n=\ell=\text { number of circumferential full waves } \\
& \tau_{K}=\text { Khot's skewedness parameter }
\end{aligned}
$$


one can reduce the solution of the linearized stability equations to an algebraic eigenvalue problem. Notice that the eigenvalue $\lambda_{m n \tau}$ depends besides on the wave numbers $m$ and $n$ also on Khot's skewedness parameter $\tau_{\mathrm{K}}$, a real number. The critical load parameter $\lambda_{c}$ is the lowest of all possible eigenvalues. Thus finding $\lambda_{c}$ involves not only a search over the integer valued wave numbers $m$ and $n$ but the search has to be repeated over a range of possible positive and negative real numbers for $\tau_{\mathrm{K}}$. Using the Level-1 computational module AXBIF [14] a search over integer valued axial half-wave numbers $\mathrm{m}$ and over a range of possible positive and negative real numbers $\tau_{\mathrm{K}}$ yielded the lowest eigenvalues listed in Table 2 for the specified circumferential wave numbers $\mathrm{n}$.

Table 2.

Buckling loads of the NASA layered

composite shell AW-CYL-1-1

Buckling load map for the perfect shell

using AXBIF [14] $\left(\mathrm{N}_{\mathrm{C} \ell}=-2238.325 \mathrm{lb} / \mathrm{in}\right)$

$$
\begin{array}{lll}
n=4 & \lambda_{c}^{m}=0.371613 & \left(m=7, \tau_{K}=3.320\right) \\
n=5 & \lambda_{c}^{m}=0.370302 & \left(m=7, \tau_{K}=2.680\right) \\
n=6 & \lambda_{c}^{m}=0.370029 & \left(m=7, \tau_{K}=2.178\right) \\
n=7 & \lambda_{c}^{m}=0.365992 & \left(m=1, \tau_{K}=0.011\right) \\
n=8 & \lambda_{c}^{m}=0.370131 & \left(m=6, \tau_{K}=1.681\right) \\
n=9 & \lambda_{c}^{m}=0.371980 & \left(m=6, \tau_{K}=1.488\right) \\
n=10 & \lambda_{c}^{m}=0.372880 & \left(m=5, \tau_{K}=1.345\right) \\
n=11 & \lambda_{c}^{m}=0.375261 & \left(m=4, \tau_{K}=1.214\right) \\
n=12 & \lambda_{c}^{m}=0.369089 & \left(m=1, \tau_{K}=0.579\right) \\
n=13 & \lambda_{c}^{m}=0.370076 & \left(m=1, \tau_{K}=0.653\right) \\
n=14 & \lambda_{c}^{m}=0.376748 & \left(m=1, \tau_{K}=0.734\right) \\
\hline & &
\end{array}
$$

Notice that besides the absolute minimum of $\lambda_{c}^{m}=0.365992$ at $n=7$ there is a local minimum of $\lambda_{c}^{m}=0.369089$ at $n=12$.

To facilitate the interpretation of the numerical results obtained, DISDECO provides the user with various graphical interfaces.

Thus the results of the search for the critical (lowest) buckling load $\lambda_{c}$ can be displayed in a contour map as shown in Fig. 1. Using membrane prebuckling the critical eigenvalue is (see also Table 2)

$$
\lambda_{\mathrm{c}}^{m}=0.365992
$$

with $m=1$ half-waves in the axial direction and $n=7$ full waves in the circumferential direction. In order to provide a quick overview of the distribution of eigenvalues, the values displayed in the contour plot are re-normalized. Thus in Fig. 1 the following re-normalized eigenvalues are plotted

$$
\rho_{c}^{m}=\frac{\lambda_{m n \tau}}{0.365992}
$$

Notice that the critical buckling load can be calculated using a simple multiplication

$$
\begin{aligned}
N_{c}^{m} & =\lambda_{c}^{m} N_{c \ell}= \\
& 0.365992(-2238.325)=-819.209 \mathrm{lb} / \mathrm{in}
\end{aligned}
$$

\section{Level-2 Perfect Shell Buckling Analysis}

To investigate the effects of edge constraint and of different boundary conditions on the critical buckling load of the perfect shell $(\bar{W}=0)$ one has to switch to the Level-2 computational module ANILISA [15]. In this module the axisymmetric prebuckling state is represented by

$$
\begin{aligned}
& W^{(0)}=h W_{v}+h W_{O}(x) \\
& F^{(0)}=\frac{E h^{2}}{c R}\left[-\frac{1}{2} \lambda y^{2}+R^{2} f_{o}(x)\right]
\end{aligned}
$$

It has been shown in Ref. [15] that with these assumptions the prebuckling problem is reduced to the solution of a single fourth order ordinary differential equation with constant coefficients, which always admits exponential solutions. Closed form solutions for simply supported and clamped boundary conditions have been published in the literature [16].

For anisotropic shells the linearized stability equations admit separable solutions of the form

$$
\begin{aligned}
& W^{(1)}=h\left[w_{1}(x) \cos n \theta+w_{2}(x) \sin n \theta\right] \\
& F^{(1)}=\frac{E R h^{2}}{c}\left[f_{1}(x) \cos n \theta+f_{2}(x) \sin n \theta\right]
\end{aligned}
$$

where $\theta=\frac{y}{R}$.

Using a generalization of Stodola's method [17] first published by Cohen [18] the resulting nonlinear eigenvalue problem is reduced to a sequence of linearized eigenvalue problems. The resulting ordinary differential equations are solved numerically by a technique known as "parallel shooting over $\mathrm{N}$-intervals" [19]. Notice 
that by this approach the effect of edge restraint and the specific boundary conditions are satisfied rigorously. To find the critical load parameter $\lambda_{c}$ an $n$-search must be carried out, whereby one must be careful to find not a local minimum but the absolute minimum. As can be seen from the results presented in Table 3 the $n$-search using membrane prebuckling and a rigorous satisfaction of SS-3 $\left(N_{X}=v=W=M_{X}=0\right)$ boundary conditions for the stability problem now yields a local minimum of $\lambda_{c}=0.364715$ at $n=7$ and an absolute minimum of $\lambda_{c}=0.364370$ at $n=11$.

Table 3

Buckling loads of the NASA layered composite shell AW-CYL-1-1 ( $\left.\mathrm{N}_{\mathrm{C} \ell}=-2238.325 \mathrm{lb} / \mathrm{in}\right)$ Buckling load map for the perfect shell using ANILISA [15] (B.C. $N_{X}=v=w=M_{X}=0$ )

\begin{tabular}{|lll|}
\hline Prebuckling: & Membrane & Nonlinear \\
$n=6$ & $\lambda_{c}^{m}=0.371920$ & $\lambda_{c}^{n l}=0.347378$ \\
$n=7$ & $\lambda_{c}^{m}=0.364715$ & $\lambda_{c}^{n l}=0.337088$ \\
$n=8$ & $\lambda_{c}^{m}=0.372592$ & $\lambda_{c}^{n l}=0.339701$ \\
$n=9$ & $\lambda_{c}^{m}=0.371460$ & $\lambda_{c}^{n l}=0.330957$ \\
$n=10$ & $\lambda_{c}^{m}=0.367479$ & $\lambda_{c}^{n l}=0.329163$ \\
$n=11$ & $\lambda_{c}^{m}=0.364370$ & $\lambda_{c}^{n l}=0.328594$ \\
$n=12$ & $\lambda_{c}^{m}=0.364551$ & $\lambda_{c}^{n l}=0.330271$ \\
$n=13$ & $\lambda_{c}^{m}=0.368528$ & $\lambda_{c}^{n l}=0.334044$ \\
$n=14$ & $\lambda_{c}^{m}=0.376314$ & $\lambda_{c}^{n l}=0.339388$ \\
\hline
\end{tabular}

The most accurate Level-2 solutions are obtained when one employs a rigorous nonlinear prebuckling analysis. As can be seen from the results listed in Table 3 , for this particular shell the critical buckling loads with nonlinear prebuckling are always lower than the corresponding results obtained using a membrane prebuckling analysis. Specifically, the local minimum of $\lambda_{C}^{n l}=0.337088$ at $n=7$ is about $8 \%$ lower, whereas the absolute minimum of $\lambda_{\mathrm{C}}^{\mathrm{nl}}=0.328594$ at $\mathrm{n}=11$ is about $11 \%$ lower. Notice that the critical load $N_{C}$ can be calculated easily by multiplying the lowest eigenvalue $\lambda_{c}$ by the normalizing factor $\mathrm{N}_{\mathrm{C} \ell}=-2238.325 \mathrm{lb} / \mathrm{in}$ yielding

$$
\mathrm{N}_{\mathrm{C}}=\lambda_{\mathrm{c}} \mathrm{N}_{\mathrm{C} \ell}=-735.500 \mathrm{lb} / \mathrm{in} \quad(\mathrm{n}=11)
$$

In fig. 2 the critical buckling modes using membrane and rigorous nonlinear prebuckling are depicted. Notice that the solutions with nonlinear prebuckling differ significantly from the ones obtained using membrane prebuckling, especially at $n=11$ where one observes a typical edge buckling type behavior.

\section{Level-3 Perfect Shell Buckling Analysis}

To verify the earlier predictions the finite difference version [20] of the well known shell analysis code STAGS [21] will be used. Due to the slightly skewed buckling pattern predicted by the Level-1 and Level-2 computations one is forced to model the whole shell.

Initially a convergence study must be carried out in order to establish the mesh size needed for accurate modeling of the buckling behavior of the shell in question. For this purpose the asymmetric bifurcation from a nonlinear prebuckling path option was used, whereby the earlier results obtained with the Level-2 module ANILISA listed in Table 3 serve as a reference.

In the convergence study, at first, for a fixed number of mesh points in the axial direction $(N R=161)$ the number of mesh points in the circumferential direction (NC) was increased until the bifurcation load approached a horizontal tangent. As can be seen from Fig. 3 the results converge to a limiting value from below at about NC $=201$. Next, for a fixed number of mesh points in the circumferential direction (NC $=201$ ) the number of rows (NR) was varied. This time convergence is from above and as can be seen from Fig. 3 the horizontal tangent is reached at about NR = 201.

Using a mesh of 161 rows and 201 columns (a model with 99268 D.O.F.'s and a maximum semi-bandwidth of 795) and SS-3 boundary conditions $\left(\mathrm{N}_{\mathrm{X}}=-\mathrm{N}_{\mathrm{O}}, \mathrm{V}=\mathrm{W}=\mathrm{M}_{\mathrm{X}}=0\right)$ the following 3 lowest eigenvalues were obtained:

$$
\begin{aligned}
& \lambda_{C}^{(1)}=0.327759 \quad(n=11) \rightarrow \\
& N_{C}^{(1)}=\lambda_{C}^{(1)} N_{C \ell}=-733.630 \mathrm{lb} / \mathrm{in} \\
& \lambda_{C}^{(2)}=0.328652 \quad(n=10) \quad \rightarrow \\
& N_{C}^{(2)}=\lambda_{C}^{(2)} N_{C \ell}=-735.631 \mathrm{lb} / \mathrm{in} \\
& \lambda_{C}^{(3)}=0.329421 \quad(n=12) \quad \rightarrow \\
& N_{C}^{(3)}=\lambda_{C}^{(3)} N_{C \ell}=-737.352 \mathrm{lb} / \mathrm{in}
\end{aligned}
$$


Details of the critical buckling mode are displayed in Fig. 4. Notice that the sequence of the 3 lowest buckling loads and the corresponding buckling modes agree closely with the predictions obtained with the Level-2 module ANILISA (see also Table 3).

\section{IMPERFECTION SENSITIVITY STUDY}

That initial imperfections may decrease the load carrying capacity of thin-walled shell structures is by now widely known and accepted. However, in order to calculate the effect of initial imperfections one must know their shape and amplitude, an information that is rarely available.

In the absence of initial imperfection measurements, as a first step one must establish whether a given shell-loading combination is imperfection sensitive, and if the answer is positive to estimate how damaging certain characteristic imperfection shapes are.

\section{Single Axisymmetric Imperfection}

Based on Koiter's pioneering work on the effect of initial imperfections $[7,22]$ the simplest imperfection model consists of a single axisymmetric imperfection

$$
\bar{W}=h \bar{\xi}_{1} \cos i \pi \frac{x}{L}
$$

where $i$ is an integer denoting the number of half-waves in the axial direction and $\bar{\xi}_{1}$ is the amplitude of the axisymmetric imperfection normalized by the shell wall-thickness $h$.

If one assumes that both the axial load and the boundary conditions are independent of the circumferential coordinate, then the prebuckling solution will also be axisymmetric, a fact that simplifies the solution considerably.

\section{Level-1 Analysis of Axisymmetric} Imperfection

Neglecting the effect of the prebuckling boundary conditions the nonlinear equations governing the prebuckling state admit the following axisymmetric solutions

$$
\begin{aligned}
& W^{(0)}=h W_{v}+h w_{0}(x) \\
& F^{(0)}=-\frac{E h^{2}}{c R} \frac{1}{2} \lambda y^{2}+f_{0}(x)
\end{aligned}
$$

where

$$
\begin{gathered}
w_{0}(x)=\frac{\lambda}{\lambda_{c_{i}}-\lambda} h \bar{\xi}_{1} \cos i \pi \frac{x}{L} \\
f_{0}(x)=\frac{\lambda}{\lambda_{c_{i}}-\lambda} \frac{\left(1+\bar{B}_{21 \alpha_{i}^{2}}^{*}\right)}{2 \alpha_{i}^{2} \bar{A}_{22}^{*}} \frac{E h^{3}}{c} \bar{\xi}_{1} \cos i \pi \frac{x}{L} \\
\lambda_{C_{i}}=\frac{1}{2}\left\{\alpha_{i}^{2} \bar{D}_{11}^{*}+\frac{\left(1+\bar{B}_{21 \alpha_{i}^{2}}^{*}\right)^{2}}{2 \alpha_{i}^{2} \bar{A}_{22}^{*}}\right\}
\end{gathered}
$$

Notice that the linearized stability equations become now a set of equations with variable coefficients. The reduced wave number $\alpha_{i}$ and the normalized stiffness coefficients $\bar{A}_{22}^{*}$, $\bar{B}_{21}^{*}$ and $\bar{D}_{11}^{*}$ are all listed in Ref. [14].

It has been shown in Ref. [14] that by assuming an asymmetric bifurcation mode of the form

$$
W^{(1)}=h \sin m \pi \frac{x}{L} \cos \frac{n}{R}\left(y-\tau_{K} x\right)
$$

where

$$
\begin{aligned}
& m=k=\text { number of axial half waves } \\
& n=\ell=\text { number of circumferential full waves } \\
& \tau_{K}=\text { Khot's skewedness parameter }
\end{aligned}
$$

a Galerkin type approximate solution yields for the eigenvalues (read, buckling loads) $\lambda$ of the problem a characteristic equation in the form of a cubic polynomial

$$
\begin{aligned}
& \lambda^{3}-\left(\lambda_{m n \tau}+2 \lambda_{c_{i}}-\hat{C}_{1} \bar{\xi}_{1} \delta_{i=2 m}\right) \lambda^{2} \\
& +\left\{2 \lambda_{m n \tau}+\lambda_{c_{i}}+\left(\hat{C_{2}}-\hat{C}_{1}\right) \bar{\xi}_{1} \delta_{i=2 m}\right\} \lambda_{c_{i}} \lambda \\
& -\left\{\lambda_{m n \tau}+\hat{C}_{2} \bar{\xi}_{1} \delta_{i=2 m}+\left(\hat{C}_{3}-\hat{C}_{4} \delta_{i m}\right) \bar{\xi}_{1}^{2}\right\} \lambda_{c_{i}}^{2}=0
\end{aligned}
$$

where

$$
\begin{aligned}
\begin{aligned}
\delta_{i=2 m}=1 \quad \text { if } i=2 m \\
=0
\end{aligned} \quad \text { otherwise } \\
\delta_{i m}=1 \quad \text { if } i=m \\
=0 \quad \text { otherwise }
\end{aligned}
$$

and the constants $\hat{C}_{1}, \hat{C}_{2}, \ldots$ are listed in Appendix $C$ of Ref. [14]. 
Here it must be remembered that one will only get any noticeable degrading influence of the assumed axisymmetric imperfection if $\bar{\xi}_{1}$ is negative and if the coupling condition $i=2 \mathrm{~m}$ is satisfied. The physical explanation for this can be found in Koiter's 1963 paper [22]. Furthermore, in order to obtain the smallest real root of Eq. (9), for a given axisymmetric imperfection $\bar{\xi}_{1}$ an $n$-search must be carried out. It should also be noticed that the terms involving the Kronecker delta $\delta_{i=2 m}$ are all linear in $\bar{\xi}_{1}$, and thus they dominate the buckling behavior of the shell with axisymmetric imperfection.

Assuming that the most likely axisymmetric imperfection of the steel mandrel used to layup the NASA composite shell AW-CYL-1-1 is given by

$$
\bar{W}=h \bar{\xi}_{1} \cos 2 \pi \frac{x}{L}
$$

the Level-1 DISDECO computational module AXBIF generated the solid curve shown in Fig. 5. Notice that the curve is re-normalized by $\lambda_{c}^{m}=0.366892$, the critical Level- 1 buckling load of the perfect shell computed using AXBIF [14] with membrane prebuckling for $\tau_{K}=0.0$ and $n=7$. Notice also that an initial imperfection amplitude equal to the wall thickness of the shell $\left(\xi_{1}=-1.0\right)$ generates a "knockdown factor" of $\rho_{c}=\lambda_{c} / \lambda_{c}^{m}=0.486$, resulting in the following rather low buckling load

$$
\begin{aligned}
& \lambda_{c}=0.486 \lambda_{c}^{m}=0.486(0.366892)=0.178310 \\
& N_{C}=\lambda_{c} N_{C \ell}= \\
& \quad 0.178310(-2238.325)=-399.115 \mathrm{lb} / \mathrm{in}
\end{aligned}
$$

with $\tau_{K}=0.0$ and $n=7$ full waves in the circumferential direction.

\section{Level-2 Analysis of Axisymmetric Imperfection}

Since the external loading, the boundary conditions and the assumed initial imperfection are axisymmetric, therefore the prebuckling solution will also be axisymmetric. It has been shown in Ref. [23] that by assuming

$$
\begin{aligned}
& W^{(0)}=h W v+h W_{0}(x) \\
& F^{(0)}=\frac{E h^{2}}{c R}\left\{-\frac{1}{2} \lambda y^{2}+R^{2} f_{O}(x)\right\}
\end{aligned}
$$

the solution of the nonlinear partial differential equations governing the prebuckling state can be reduced to the solution of a single fourth order ordinary differential equation with constant coefficients, which can be solved routinely.

For anisotropic shells the resulting linearized stability equations admit separable solutions of the form

$$
\begin{aligned}
& W^{(1)}=h\left[w_{1}(x) \cos n \theta+w_{2}(x) \sin n \theta\right] \\
& F^{(1)}=\frac{E R h^{2}}{c}\left[f_{1}(x) \cos n \theta+f_{2}(x) \sin n \theta\right]
\end{aligned}
$$

where $\theta=\frac{y}{R}$.

Solution proceeds as outlined on Ref. [23]. Using an updated version of the Level-2 computational module ANILISA [24] and SS-3 $\left(N_{X}=v=W=M_{X}=0\right)$ boundary conditions one obtains the results presented in Table 4. Notice that a rigorous nonlinear prebuckling analysis was used and an n-search was carried out for each specified axisymmetric imperfection amplitude $\bar{\xi}_{1}$.

The values of Table 4 are plotted as the dashed curve in Fig. 5. A comparison of the results obtained via the Level-1 module AXBIF (solid curve) and the Level-2 module ANILISA (dashed curve) shows that also in the case of axisymmetric imperfections a rigorous prebuckling analysis should be used. Especially for very small initial imperfection amplitudes $\left(\left|\bar{\xi}_{1}\right|<0.1\right)$ the Level-1 predictions are inaccurate and overestimate the critical buckling load. Notice further that both curves have been normalized by $\lambda_{c}^{m}=0.366892$, the critical Level-1 buckling load of the perfect shell computed using membrane prebuckling by AXBIF [14] for $\tau_{K}=0.0$ and $n=7$. This way the effect of using a rigorous prebuckling analysis becomes easily discernible.

It is interesting to see that for small enough initial imperfection amplitudes $\left(\left|\bar{\xi}_{1}\right|<0.07\right.$, say) the critical buckling load of the shell is insensitive to the initial imperfection shape specified by Eq. (10). Notice that the critical buckling modes have $n=11$ full waves in the circumferential direction, and as can be seen in Fig. 6, a somewhat skewed buckling mode shape which is dominated by edge buckling. However, larger initial imperfections $\left(\left|\bar{\xi}_{1}\right| \geq 0.07\right.$, say) force the shell to respond in another mode shape with $n=7$ full waves in 
the circumferential direction and with practically straight nodal lines. Interestingly enough now the critical buckling load of the shell is sensitive to the axisymmetric initial imperfection shape specified by Eq. (10) and an initial imperfection amplitude equal to the wall thickness of the shell $\left(\bar{\xi}_{1}=-1.0\right)$ generates a "knockdown factor" of $\rho_{c}=\lambda_{c} / \lambda_{C}^{m}=0.418$. It predicts thus the following rather low buckling load

$$
\begin{aligned}
& \lambda_{\mathrm{C}}=0.418 \lambda_{\mathrm{c}}^{\mathrm{m}}=0.418(0.366892)=0.153361 \\
& \mathrm{~N}_{\mathrm{C}}=\lambda_{\mathrm{c}} \mathrm{N}_{\mathrm{C} \ell}= \\
& \quad 0.153361(-2238.325)=-343.271 \mathrm{lb} / \mathrm{in}
\end{aligned}
$$

with a very slight skewedness of the buckling pattern and $n=7$ full waves in the circumferential direction.

Table 4

Buckling loads of the NASA layered composite shell AW-CYL-1-1 $\left(\mathrm{N}_{\mathrm{C} \ell}=-2238.325 \mathrm{lb} / \mathrm{in}\right)$

Axisymmetric imperfection using ANILISA [24]

(B.C. : $N_{x}=v=w=M_{X}=0$ )

\begin{tabular}{|l|l|l|l|}
\hline $\bar{\xi}_{1}$ & $\lambda_{c}^{n l}$ & $\bar{\xi}_{1}$ & $\lambda_{c}^{\mathrm{nl}}$ \\
\hline 0. & $0.328594(n=11)$ & -0.2 & $0.299830(n=7)$ \\
-0.01 & $0.328671(n=11)$ & -0.3 & $0.274773(n=7)$ \\
-0.02 & $0.328745(n=11)$ & -0.4 & $0.251275(n=7)$ \\
-0.05 & $0.328939(n=11)$ & -0.5 & $0.229976(n=7)$ \\
-0.06 & $0.328994(n=11)$ & -0.6 & $0.210876(n=7)$ \\
-0.07 & $0.328332(n=7)$ & -0.7 & $0.193827(n=7)$ \\
-0.08 & $0.326652(n=7)$ & -0.8 & $0.178655(n=7)$ \\
-0.09 & $0.324860(n=7)$ & -0.9 & $0.165187(n=7)$ \\
-0.10 & $0.322960(n=7)$ & -1.0 & $0.153263(n=7)$ \\
\hline
\end{tabular}

\section{Level-3 Analysis of Axisymmetric} Imperfection

Recalling that since both the axial load and the boundary conditions are independent of the circumferential coordinate, therefore the prebuckling solution will also be axisymmetric, one can use once again the asymmetric bifurcation from a nonlinear prebuckling path option. By modeling the full shell the code can choose itself the critical number of full waves in the circumferential direction. No n-search must be carried out. Using a uniformly spaced mesh of 161 rows and 201 columns and the user written subroutine option WIMP to introduce the following axisymmetric imperfection

$$
\bar{W}=1.0 h \cos 2 \pi \frac{x}{L}
$$

(remember STAGS defines $\bar{W}$ positive outward) the following critical bifurcation load was found

$$
\mathrm{N}_{\mathrm{C}}=-333.449 \mathrm{lb} / \mathrm{in}
$$

As can be seen from Fig. 7 the critical buckling mode has $n=7$ full waves in the circumferential direction and no visible skewedness. The nondimensional bifurcation load of the shell with axisymmetric imperfection is for $\bar{\xi}_{1}=1.0$

$$
\lambda_{c}=\frac{N_{c}}{N_{C \ell}}=\frac{-333.449}{-2238.325}=0.148973
$$

The re-normalized bifurcation load is

$$
\rho_{\mathrm{C}}^{\mathrm{nl}}=\frac{\mathrm{N}_{\mathrm{C}}}{\overline{\mathrm{N}}_{\mathrm{C} \ell}} \text { where } \overline{\mathrm{N}}_{\mathrm{C} \ell}=\lambda_{\mathrm{C}}^{\mathrm{nl}} \mathrm{N}_{\mathrm{C} \ell}
$$

thus

$$
\rho_{c}^{n l}=\frac{\lambda_{c}}{\lambda_{c}^{n l}}=\frac{0.148973}{0.327759}=0.4545
$$

Notice that the Level-3 re-normalization is done using $\lambda_{c}^{n l}=0.327759$, the critical Level-3 buckling load of the perfect shell computed using STAGS-A with nonlinear prebuckling and a $161 \times 201$ mesh.

Notice also that the Level-2 ANILISA prediction $\left(\rho_{\mathrm{C}}^{\mathrm{nl}}=0.4664, n=7\right)$ agrees closely with the Level-3 STAGS-A prediction $\left(\rho_{C}^{n !}=0.4545\right.$, $n=7$ ). The slight difference is partly due to the fact that ANILISA uses the Donnell type nonlinear shell equations, whereas STAGS-A employs the higher order Marlowe-Flügge equations.

\section{Single Asymmetric Imperfection}

The effect of a single asymmetric initial imperfection can be investigated either by solving the full nonlinear response problem or by employing the well known LyapunovSchmidt-Koiter [7] reduction technique. When investigating the degrading effect of a single mode asymmetric imperfection

$$
\bar{W}=t \bar{\xi}_{2} \sin m \pi \frac{x}{L} \cos n \frac{y}{R}
$$

where $m$ and $n$ are integers denoting the number of axial half-waves and the number of circumferential full waves, respectively, 
instability occurs at the limit point of the prebuckling state in the generalized loaddeformation space. Assuming that the eigenvalue problem for the critical (lowest) buckling load $\Lambda_{c}$ will yield a unique asymmetric buckling mode $W^{(1)}$, then for an imperfect shell $\left(\bar{\xi}_{2} \neq 0\right)$ the shape of the generalized load-deflection curve in the vicinity of the bifurcation point $\Lambda=\Lambda_{c}$ is given by the following asymptotic expansion

$$
\begin{aligned}
& \left(\Lambda-\Lambda_{\mathrm{c}}\right) \xi=\Lambda_{\mathrm{c}} \mathrm{a} \xi^{2}+\Lambda_{\mathrm{c}} \mathrm{b} \xi^{3}+\ldots \\
& -\Lambda_{\mathrm{c}} \alpha \bar{\xi}_{2}-\left(\Lambda-\Lambda_{\mathrm{c}}\right) \beta \bar{\xi}_{2}+\mathrm{O}\left(\xi \bar{\xi}_{2}\right)
\end{aligned}
$$

Expressions for the postbuckling coefficients " $a$ " and " $\mathrm{b}$ " and the imperfection forms factors " $\alpha$ " and " $\beta$ " are derived in References [25,26]. If the limit point is close to the bifurcation point, then the maximum load $\Lambda_{S}$ that the structure can carry prior to buckling can be evaluated from Eq. (14) by maximizing $\Lambda$ with respect to $\xi$. For cases where the first postbuckling coefficient "a" is zero, this analysis yields the modified Koiter formula [26]

$$
\left(1-\rho_{s}\right)^{3 / 2}=\frac{3}{2} \sqrt{-3 \alpha^{2} b}\left[1-\frac{\beta}{\alpha}\left(1-\rho_{s}\right)\right]\left|\bar{\xi}_{2}\right|(15)
$$

where $\rho_{\mathrm{s}}=\Lambda_{\mathrm{s}} / \Lambda_{\mathrm{c}}$.

Notice that, if the second postbuckling coefficient " $b$ " is positive, Eq. (15) has no real solutions. Thus the buckling load of the specified shell-loading combination is not sensitive to small asymmetric initial imperfections of the shape given by Eq. (13). If, however, the second postbuckling coefficient " $b$ " is negative, the equilibrium load $\Lambda$ decreases following buckling and the buckling load of the real structure $\Lambda_{S}$ is sensitive to the asymmetric initial imperfection specified by Eq. (13).

\section{Level-1 Analysis of Asymmetric} Imperfection

For the composite shell under investigation, as can be seen from the partial results listed in Table 2, there are many eigenvalues only slightly higher than the critical one of $\lambda_{c}=0.365992$ for $m=1, n=7$ and $\tau_{\mathrm{K}}=0.011$. Hence, strictly speaking, the proposed form of the perturbation expansion given by Eqs. (14) is not applicable, since the nonlinear interaction between the many nearly simultaneous eigenmodes is not accounted for. Thus the following results, where one considers the eigenfunctions corresponding to certain critical eigenvalues chosen one at the time, can at best give an indication as to the severity of the expected imperfection sensitivity.

Assuming initially an asymmetric imperfection affine to the critical buckling mode of the perfect NASA composite shell AW-CYL1-1 as computed by the Level-1 computational module AXBIF (see also Table 2)

$$
\bar{W}=h \bar{\xi}_{2} \sin \pi \frac{x}{L} \cos \frac{7}{R}(y-0.011 x)
$$

and using the Level-1 computational module BFACT to carry out the initial postbuckling analysis yields the following results

$$
\begin{aligned}
& \lambda_{C}=\lambda_{c}^{m}=0.365992\left(m=1, n=7, \tau_{K}=0.011\right) \\
& b=-0.048844 \quad \alpha=\beta=1.0
\end{aligned}
$$

Substituting these values into Eq. (15), one can plot the degrading effect of an asymmetric imperfection of the shape given by Eq. (16) as a function of its amplitude $\bar{\xi}_{2}$. As can be seen from Fig. 8 an initial imperfection amplitude equal to the wall thickness of the shell $\left.\bar{\xi}_{2}=1.0\right)$ generates a "knockdown factor" of $\rho_{S}=\lambda_{S} / \lambda_{C}^{m}=0.541$, resulting in the following rather low buckling load

$$
\begin{aligned}
\lambda_{S}= & 0.541 \lambda_{C}^{m}=0.541(0.365992)=0.198002 \\
N_{S}= & \lambda_{S} N_{C \ell}= \\
& 0.198002(-2238.325)=-443.192 \mathrm{lb} / \mathrm{in}
\end{aligned}
$$

Notice that the imperfection form factors " $\alpha$ " and " $\beta$ " are identical equal to 1.0 because BFACT uses membrane prebuckling to calculate the necessary first and second order fields and the assumed asymmetric imperfection shape of Eq. (16) is affine to the buckling mode. Please notice that in Fig. 8 the collapse load is re-normalized by $\lambda_{c}^{m}=0.365992$, the critical Level- 1 buckling load of the perfect shell computed using membrane prebuckling by AXBIF [14] for $\tau_{K}=0.011$ and $n=7$.

\section{Level-2 Analysis of Asymmetric Imperfection}

To investigate the effects of edge-constraint and/or different boundary conditions on the imperfection sensitivity of the critical buckling load of the NASA composite shell AW-CYL-1-1 
one has to switch to the Level-2 module ANILISA [15] and run its postbuckling analysis option. In this module, as described earlier, the axisymmetric prebuckling state is represented by Eqs. (3), the buckling modes by Eqs. (4) and the postbuckling state by

$$
\begin{aligned}
& W^{(2)}=h\left[w_{\alpha}(x)+w_{\beta}(x) \cos n \theta+w_{\gamma}(x) \sin n \theta\right] \\
& F^{(2)}=\frac{E R h^{2}}{c}\left[f_{\alpha}(x)+f_{\beta}(x) \cos 2 n \theta+f_{\gamma}(x) \sin 2 n \theta\right]
\end{aligned}
$$

where $\theta=y / R$. Details of the computational procedures used are reported in Refs. $[15,23]$.

Next, let us assume that the specified asymmetric imperfection is affine to the critical buckling mode obtained by the rigorous Level2 perfect shell analysis discussed earlier

$$
\bar{W}=h \bar{\xi}_{2}\left[w_{1}(x) \cos 11 \theta+w_{2}(x) \sin 11 \theta\right]
$$

where $\theta=y / R$ and the component functions $w_{1}(x)$ and $w_{2}(x)$ are shown in Fig. $2 b$. Running ANILISA with rigorous prebuckling and SS- 3 boundary conditions $\left(\mathrm{N}_{\mathrm{X}}=-\mathrm{N}_{\mathrm{O}}\right.$, $v=w=M_{X}=0$ ) yields the following results

$$
\begin{aligned}
& \lambda_{c}=\lambda_{C}^{n \ell}=0.328594(n=11) \\
& b=-0.37605 ; \alpha=0.46663 ; \beta=-0.22174
\end{aligned}
$$

Using Eq. (15) to plot the degrading effect of the asymmetric imperfection specified by Eq. (18) as a function of its amplitude $\bar{\xi}_{2}$ one obtains the results displayed in Fig. 9 as a solid line. Obviously the fact that for an imperfection shape affine to (similar to) the buckling mode with an amplitude of $\bar{\xi}_{2}=1.0$ one obtains a negative load carrying capacity is unrealistic.

Here one must remember that Koiter's Sensitivity Theory is asymptotically exact, that is, it yields accurate predictions for sufficiently small imperfections, whereby what is sufficiently small may vary from case to case. Also, Eq. (15) was obtained by using the perturbation expansion given by Eq. (14), where terms of order $(\bar{\xi} \bar{\xi})$ are neglected. As can be seen from the dotted curve plotted in Fig. 9 , by using more advanced computational modules such as COLLAPSE [27], where a full nonlinear solution is used and terms up to and including order $\left(\xi \bar{\xi}^{2}\right)$ are kept, one obtains more reasonable predictions.
Notice that up to about $\bar{\xi}_{2}=0.3$ the asymptotic predictions from ANILISA and the nonlinear results of COLLAPSE agree very closely. Thus one can say that in this case the range of validity of the asymptotic solution is $0 \geq \bar{\xi}_{2} \geq 0.3$.

\section{DISCUSSION OF THE RESULTS}

When comparing and analyzing the results obtained sofar it is important to keep in mind that all Level-1 and Level-2 solutions are based on approximate representations of the unknown functions. As pointed out in the previous sections Level-1 solutions use a single term double Fourier series approximation to reduce the solution of the stability problem, formulated in terms of partial differential equations, to algebraic eigenvalue problems. The effect of edge restraint is neglected (one uses a membrane prebuckling solution) and the assumed field functions satisfy approximately SS-3 $\quad\left(\mathrm{N}_{\mathrm{X}}=-\mathrm{N}_{\mathrm{O}}\right.$. $v=w=M_{X}=0$ ) boundary conditions.

Level-2 solutions eliminate the $y$ dependence by a truncated Fourier decomposition in the circumferential direction. The resulting system of nonlinear ordinary differential equations are solved numerically, whereby both the specified boundary conditions and the effect of edge restraint are rigorously satisfied. Thus by this approach the only approximation is that one represents the variation of the solution in the circumferential direction by a single harmonic with $n$ full waves, whereby an $n$-search is used to establish which wave number is the critical one. The Level-2 module ANILISA can also be used to investigate the effect of using different boundary conditions. In Table 5, the results for four different boundary conditions are presented. As expected the fully clamped C4 boundary conditions has the highest critical buckling load. The increase in load carrying capacity with respect to the weaker SS3 boundary conditions is about the same as for an isotropic shell of similar characteristic dimensions (same L/R and R/t ratios) of Ref. [28].

The Level-3 solutions are based either on a 2-dimensional finite difference or finite element formulation. In both cases, if one uses the appropriate meshes, one can obtain rigorous solutions where all nonlinear effects are properly accounted for. The only real problem with Level-3 type solutions is that for each problem one must establish the appropriate mesh size. Coarse meshes yield inaccurate solutions. What is coarse depends on the particular problem under investigation. Thus, 
for a general nonlinear solution a convergence study must always be carried out.

Using a hierarchical simulation platform such as DISDECO (Delft Interactive Shell DEsign Code), where the analyst has at his disposal computational modules of different level of sophistication, such a convergence study can be carried out relatively quickly and accurately. In Table 5 a summary of the results obtained in this study is presented using normalized variables. In Table 6 the same results are repeated but this time the imperfect buckling loads are printed as re-normalized variables $\rho$. Looking at the first column, where the critical buckling loads and the critical buckling mode shapes of the perfect shell are listed, one sees that using the Level-3 code STAGS-A one must indeed use a relatively fine mesh (161 rows and 201 columns) in order to obtain an accurate prediction of the critical buckling load. Remember, all preceding computational modules are based on Donnell type anisotropic shell equations, however, STAGS-A uses the more accurate MarloweFlügge type equations. Usually the use of a more refined theory implies a lower buckling load. The value of the normalization factor used, $\mathrm{N}_{\mathrm{C} \ell}=\mathrm{Eh}^{2} / \mathrm{cR}$, is printed in the heading of the table. The lowest critical buckling load was found using STAGS-A and modeling the whole shell with a mesh consisting of 161 rows and 201 columns. The asymmetric bifurcation from a nonlinear prebuckling path option with SS-3 boundary conditions yielded

$$
\begin{aligned}
N_{C}^{n l}=0.327759 & (-2238.325) \\
& =-733.630 \mathrm{lb} / \mathrm{in}(n=11)
\end{aligned}
$$

Considering now the effect of different types of imperfection shapes a second normalization is introduced, whereby the new normalization factors are chosen such that for vanishingly small imperfections the normalized variable $\rho$ approaches unity (i.e. 1.000). The only exception to this rule is the case of the axisymmetric imperfection

$$
\bar{W}=-h \bar{\xi}_{1} \cos 2 \pi \frac{x}{L}
$$

where by using as the normalization factor $\lambda_{c}^{*}=0.366892$ (the asymmetric bifurcation perfect shell buckling load with membrane prebuckling, $\tau_{K}=0.0$ and $n=7$ ) in the limit as $\bar{\xi}_{1} \rightarrow 0, \rho_{\mathrm{C}}$ approaches the value

$$
\rho_{\mathrm{C}}=\frac{\lambda_{\mathrm{C}}}{\lambda_{\mathrm{C}}^{*}}=\frac{0.328594}{0.366892}=0.895615 \sim 0.896
$$

This value represents the effect of edge restraint. See also the results of Table 4 and Fig. 5. Notice that using membrane prebuckling, thus neglecting the effect of edge restraint, the normalized buckling load of the perfect shell is 1.000 .

Turning now to the effect of asymmetric imperfections, from the results listed in Table 5 it is evident that for an imperfection amplitude equal to one wall-thickness the range of validity of the asymptotic solutions is exceeded, and the predictions of Koiter's imperfection sensitivity theory computed by ANILISA [15] are no longer valid (see also Fig. 9). On the other hand, there seems to be good agreement between the results obtained by COLLAPSE [27], a Level-2 computational module which computes a nonlinear solution based on a two modes approximation, and the STAGS-A [20] solution obtained sofar for the two modal imperfections and the affine imperfection listed in Table 5 . In general the more accurate STAGS-A, Level-3 solutions are slightly lower than the Level-2 COLLAPSE solutions, with exception of the asymmetric imperfection affine to the perfect shell buckling mode. The reason for this anomaly lies in the fact that this imperfection triggers more than one circumferential harmonic close to the limit point (as can be seen in Fig. 66 of Ref. 29).

\section{CONCLUSIONS}

By relying on a series of theoretical results of various degree of sophistication published in the literature, the hierarchical approach used in this paper has resulted in a series of buckling load predictions of increasing accuracy. It was shown that in order to be able to arrive at a reliable prediction of the critical buckling load and to make an estimate of its imperfection sensitivity which can be used with confidence, one must proceed step by step from simple to more complex models and solution procedures.

In particular one can state, that in order to predict the critical buckling load accurately and to make a reliable estimate of its imperfection sensitivity, the nonlinear effects caused by the edge restraint conditions must be included in the analysis. Any solution procedure which fails to account for these effects, should be suspect of having provided incorrect results.

The most approximate of the here described analyses, the Level- 1 solutions which neglect the effects caused by the edge restraints, can still be used to great advantage 
to establish the approximate behavior of a shell subjected to the specified external loading. However, depending on the value of the prebuckling stiffness, resulting from the different types of wall constructions used, the solutions may be either conservative or nonconservative.

As can be seen from the results shown in Tables 5 and 6 , the buckling load of the composite shell AW-CYL-1-1 is sensitive to all the initial imperfection shapes investigated. For a more specific prediction of the final collapse load, the final goal of a "High Fidelity Buckling Load Analysis", one would have to carry out a refined Level-3 analysis including measured values of all the significant generalized imperfections such as the traditional shell-wall imperfections, variations in the shell-end or loading surface geometry and especially for composite shells variations in the shell-wall thickness distribution. It has been shown in Ref. 30 that such an approach yields very good agreement between the predicted collapse load and the experimental buckling load. Such a paper is in preparation.

\section{ACKNOWLEDGEMENT}

The research reported in this paper was supported in part by NASA Grant NAG 1-2129. This aid is gratefully acknowledged.

\section{REFERENCES}

1. Waters, W.A. Jr., "Effects of Initial Geometric Imperfections on the Behavior of Graphite-Epoxy Cylinders Loaded in Compression", M.Sc. Thesis in Engineering Mechanics, Old Dominion University, Norfolk, Virginia, December 1996.

2. Arbocz, J., Starnes, J.H. and Nemeth, M.P., "A Hierarchical Approach to Buckling Load Calculations", Proceedings 40th AIAAASME/ASCE/AHS/ASC Structures, Structural Dynamics and Materials Conference, April 12-15, 1999, St. Louis, MO, pp. 289-299.

3. Anonymous, NASTRAN, The MacNealSchwendler Corporation, Los Angeles, California.

4. Anonymous, ABAQUS, Hibbitt, Karlsson \& Sorensen, Inc., Providence, Rhode Island.

5. Zienkiewicz, O.C., "The Finite Element Method", (The third, expanded and revised edition of The Finite Element Method in Engineering Science), McGraw-Hill Book Company (UK) Limited, London, 1977, p. 504.
6. Byskov, E., "Smooth Postbuckling Stresses by a Modified Finite Element Method", DCAMM Report No. 380, Technical University of Denmark, Lyngby, 1988.

7. Koiter, W.T., "On the Stability of Elastic Equilibrium", Ph.D. Thesis (in Dutch), THDelft, The Netherlands, H.J. Paris, Amsterdam, 1945, English translation NASA TTF-10, 1967, pp. 1-833.

8. Budiansky, B. and Hutchinson, J.W., "Dynamic Buckling of Imperfection Sensitive Structures", Proceedings 11th IUTAM Congress, Munich, 1964, Julius Springer Verlag, Berlin, 1966, pp. 636651.

9. Hoff, N.J., "Buckling of Thin Shells", Proceedings of an Aerospace Symposium of Distinguished Lecturers in Honor of Theodore von Kármán on his 80th Anniversary, Institute of Aerospace Sciences, New York, 1961, pp. 1-42.

10. Stuhlman, C.E., De Luzio, A. and Almroth, B., "Influence of Stiffener Eccentricity and End Moment on Stability of Cylinders in Compression", AIAA Journal, Vol. 4, No. 5, May 1966, pp. 872-877.

11. Fischer, G., "Über den Einfluss der gelenkingen Lagerung auf die Stabilität dünnwandiger Kreiszylinderschalen unter Axiallast und Innendruck", Z.f. Flugwissenschaften, Vol. 11, 1963, pp. 111119.

12. Stein, M., The Influence of Prebuckling Deformations and Stresses on the Buckling of Perfect Cylinders", NASA TR190, February 1964.

13. Arbocz, J. and Hol, J.M.A.M., "Shell Stability Analysis in a Computer-Aided Engineering (CAE) Environment", Proceedings 34th AIAA/ASME/ASCE/ AHS/ASC Structures, Structural Dynamics and Materials Conference, April 19-22, 1993, La Jolla, California, pp. 300-314.

14. Arbocz, J., "The Effect of Initial Imperfections on Shell Stability - An Updated Review", Report LR-695, Delft University of Technology, Faculty of Aerospace Engineering, The Netherlands, September 1992.

15. Arbocz, J. and Hol, J.M.A.M., "Koiter's Stability Theory in a Computer Aided Engineering (CAE) Environment", Int. J. Solids and Structures, Vol. 26, No. 9/10, pp. 945-973, 1990.

16. Booton, M., "Buckling of Imperfect Anisotropic Cylinders under Combined Loading", UTIAS Report No. 203, University of Toronto, 1976.

17. Von Kármán, T. and Biot, M.A., "Mathematical Methods in Engineering", 
McGraw-Hill Book Co., New York, London, 1940, p. 317.

18. Cohen, G.A., "Computer Analysis of Asymmetric Buckling of Ring-stiffened Orthotropic Shells of Revolution", AIAA Journal, Vol. 6, No. 1, January 1968, pp. 141-149.

19. Keller, H., "Numerical Methods of TwoPoint Boundary Value Problems", Blaisdell Publishing Co., Waltham, Mass., 1968.

20. Almroth, B.O., Brogan, F.A., Miller, E., Zele, F. and Peterson, H.T., "Collapse Analysis for Shells of General Shape; II. User's Manual for the STAGS-A Computer Code", Technical Report AFFDL-TR-71-8, Air Force Flight Dynamics Laboratory, Wright-Patterson Air Force Base, Ohio, March 1973.

21. Brogan, F.A., Rankin, C.C. and Cabiness, H.D., "STags Access Routines-STAR Reference Manual", Report LMSC P032595, Version 2.0, Lockheed Palo Alto Research Laboratory, Palo Alto, California, June 1994.

22. Koiter, W.T., "The Effect of Axisymmetric Imperfections on the Buckling of Cylindrical Shells under Axial Compression", Koninkl. Ned. Akad. Wetenschap. Proc. B66, 1963, pp. 265-279.

23. Arbocz, J. and Hol, J.M.A.M., "ANILISA Computational Module for Koiter's Imperfection Sensitivity Theory", Report LR-582, Delft University of Technology, Faculty of Aerospace Engineering, The Netherlands, January 1989.

24. Romkes, A., "Stability and Imperfection Sensitivity of Anisotropic Cylindrical Shells under General Boundary Conditions", Memorandum M-809, Delft University of Technology, Faculty of Aerospace Engineering, The Netherlands, July 1997.

25. Hutchinson, J.W. and Amazigo, J.C., "Imperfection Sensitivity of Eccentrically Stiffened Cylindrical Shells," AIAA Journal, Vol. 5, No. 3, March 1967, pp. 392-401.

26. Cohen, G.A., "Effect of a Nonlinear Prebuckling State on the Postbuckling Behavior and Imperfection Sensitivity of Elastic Structures," AIAA Journal, Vol. 6, No. 8, August 1968, pp. 1616-1619.

27. Arbocz, J., de Vries, J. and Hol, J.M.A.M., "On the Buckling of Imperfect Anisotropic Shells with Elastic Edge Supports under Combined Loading - Part I: Theory and Numerical Analysis", Memorandum M-849, Delft University of Technology, Faculty of Aerospace Engineering, The Netherlands, May 1997.

28. Almroth, B.O., "Influence of Edge Conditions on the Stability of Axially
Compressed Cylindrical Shells", NASA CR-161, February 1965.

29. Arbocz, J., "Critical Buckling Load and Imperfection Sensitivity Calculations for an Axially Compressed Composite Shell with Symmetrical Lay-up", Memorandum M791, Delft University of Technology, Faculty of Aerospace Engineering, The Netherlands, March 1997.

30. Hilburger, M.W. and Starnes, J.H. Jr., "Effects of Imperfections on the Buckling Response of Compression-Loaded Composite Shells", Proceedings 41st AIAA/ASMEJASCE/AHS/ASC Structures, Structural Dynamics and Materials Conference, 3-6 April 2000, Atlanta, Georgia, (paper AIAA-2000-1382). 


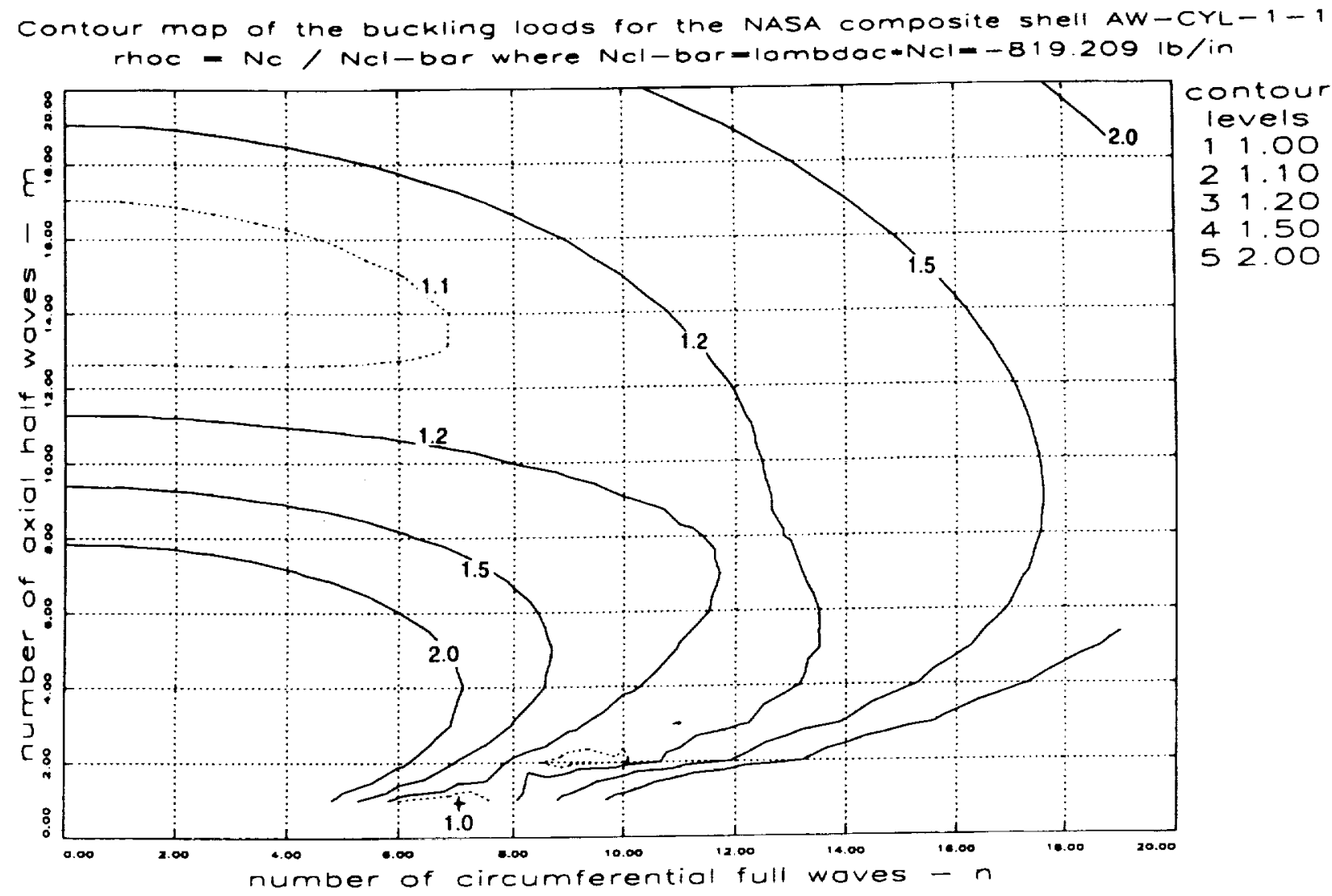

Fig. 1 Distribution of buckling loads based on Level-1 membrane prebuckling analysis - NASA composite shell AW-CYL-1-1 


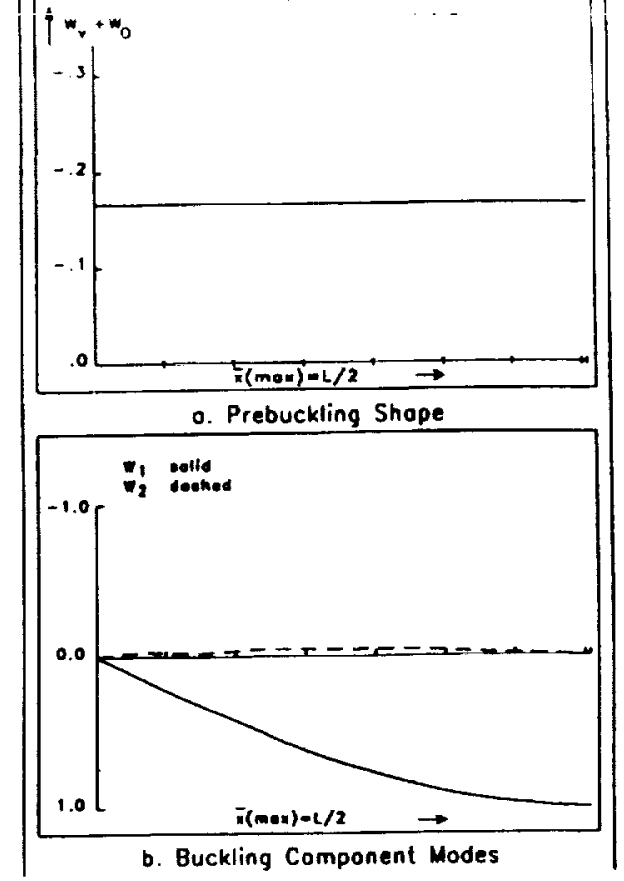

a. Membrane prebuckling, $n=7$

$\lambda_{\mathrm{C}}^{\mathrm{m}}=0.364715$

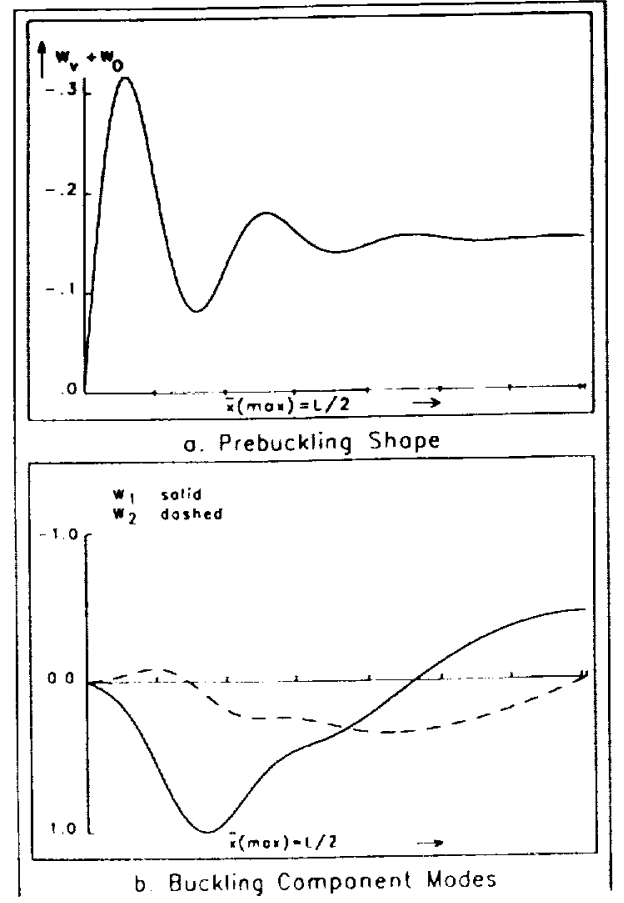

b. Nonlinear prebuckling, $n=11$ $\lambda_{c}^{n l}=0.328594$

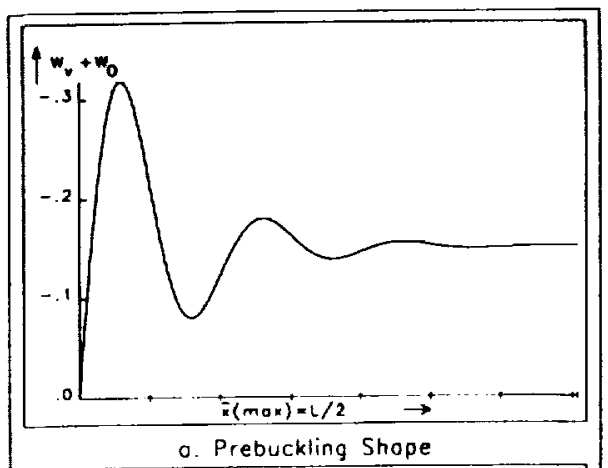

Wotid

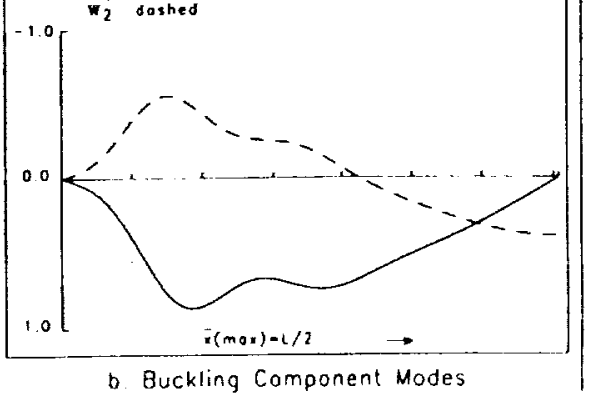

c. Nonlinear prebuckling, $\mathrm{n}=10$ $\lambda_{\mathrm{c}}^{\mathrm{nl}}=0.329163$

Fig. 2 Buckling modes of the axially compressed layered composite shell AW-CYL-1-1 SS-3 B.C. $-N_{x}=-N_{0}, v=W=M_{x}=0 ; N_{c \ell}=-2238.325 \mathrm{lb} / \mathrm{in}$ 


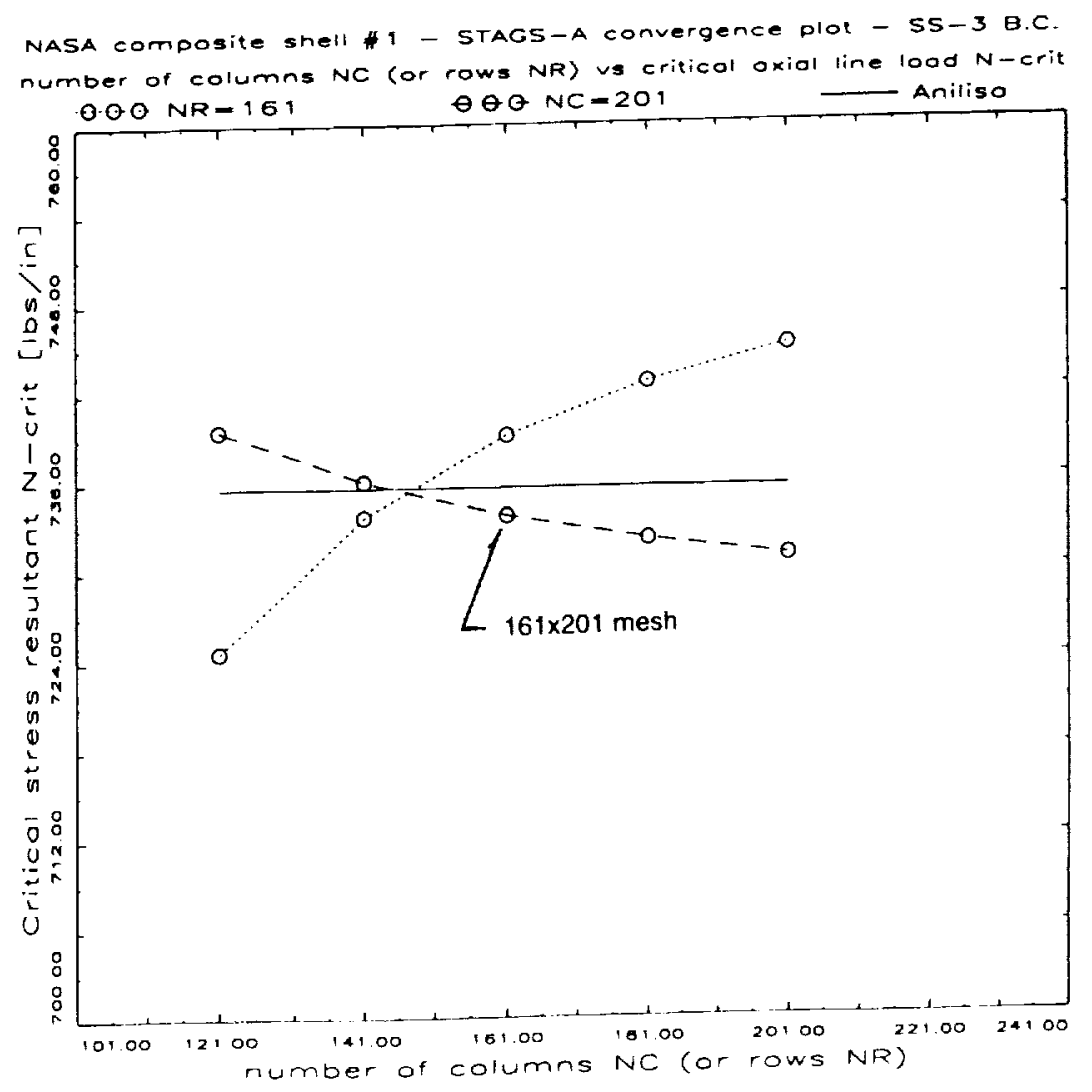

Fig. 3 STAGS-A convergence study - NASA composite shell AW-CYL-1-1 


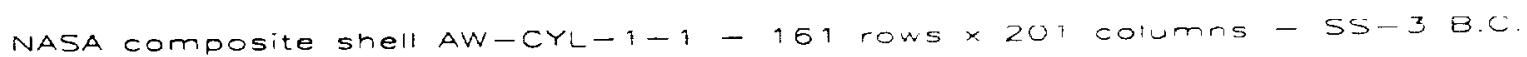
critical buckling mode - w field - Nc=-733.640 lb/in - delto-teta=360 degrees

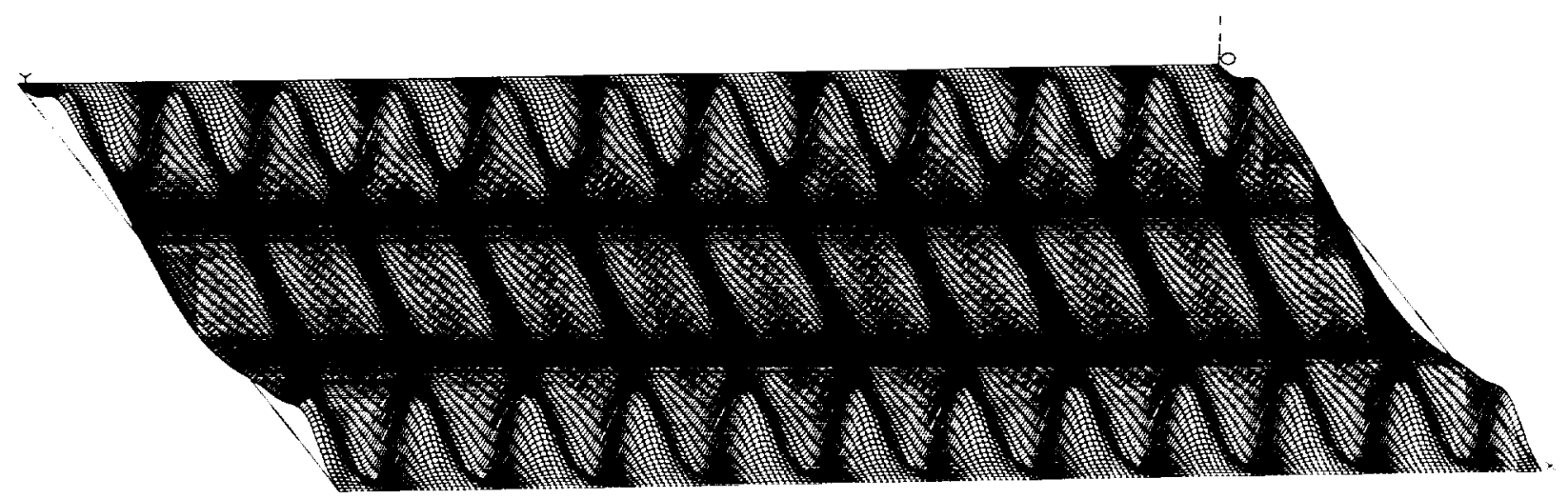

a. General view of the critical buckling mode

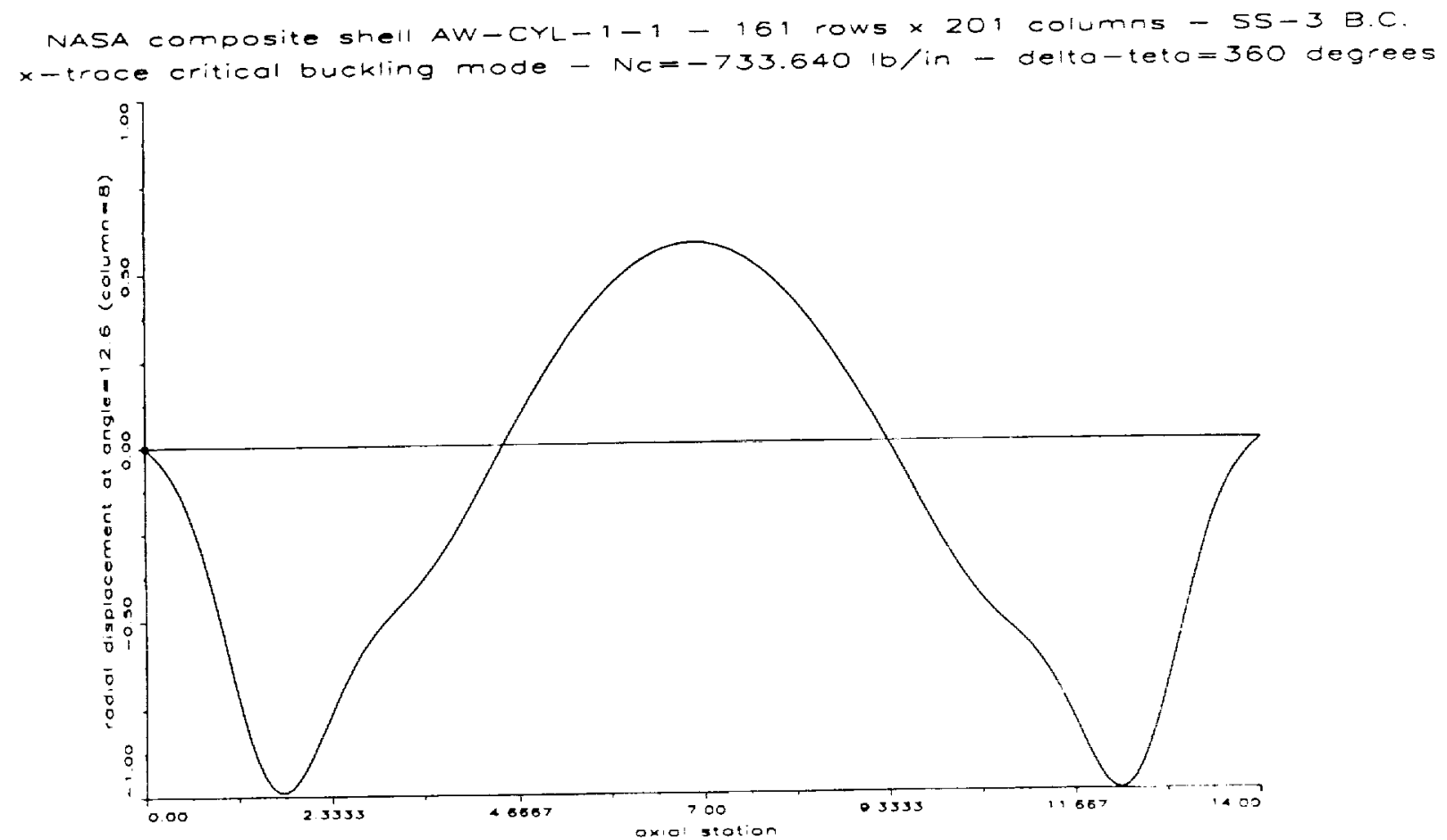

b. Axial trace of the critical buckling mode

Fig. 4 Buckling mode of the axially compressed layered composite shell AW-CYL-1-1 SS-3 B.C. $-N_{x}=-N_{0}, V=W=M_{x}=0 ; N_{C}=-2238.325 \mathrm{lb} / \mathrm{in} ;$ STAGS-A results 
NASA composite shell AW-CYL-1-1-161 rows 1 - 201 columns - SS-3 B.C.

critical buckling mode ot $N C=-333.449$ lb/in - Woor/h=1.0*cos(2*pi*x/L)

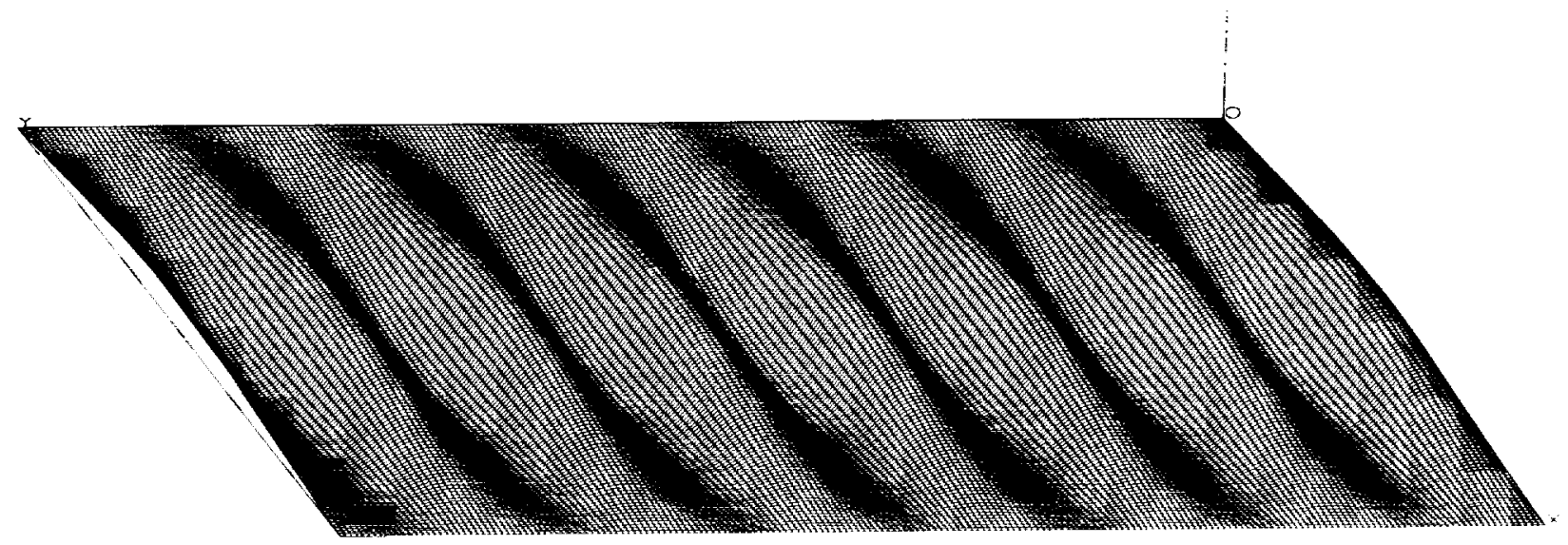

Fig. 7 General view of the critical buckling mode - axisymmetric imperfection

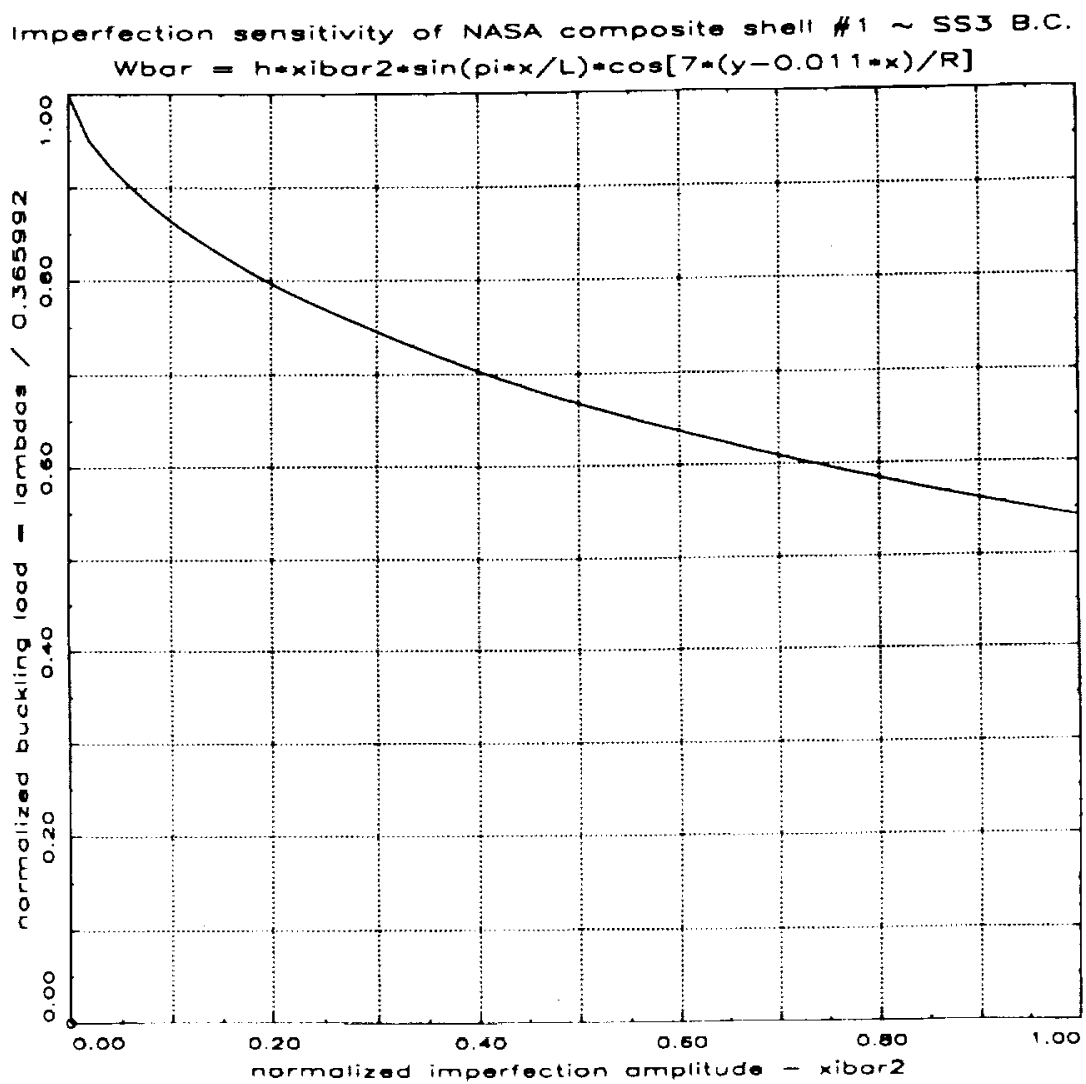

Fig. 8 Level-1 imperfection sensitivity calculation using BFACT [14] Asymptotic theory: $\bar{W}=h \bar{\xi}_{2} \sin \pi \frac{x}{L} \cos \frac{7}{R}(y-0.011 x)$ 


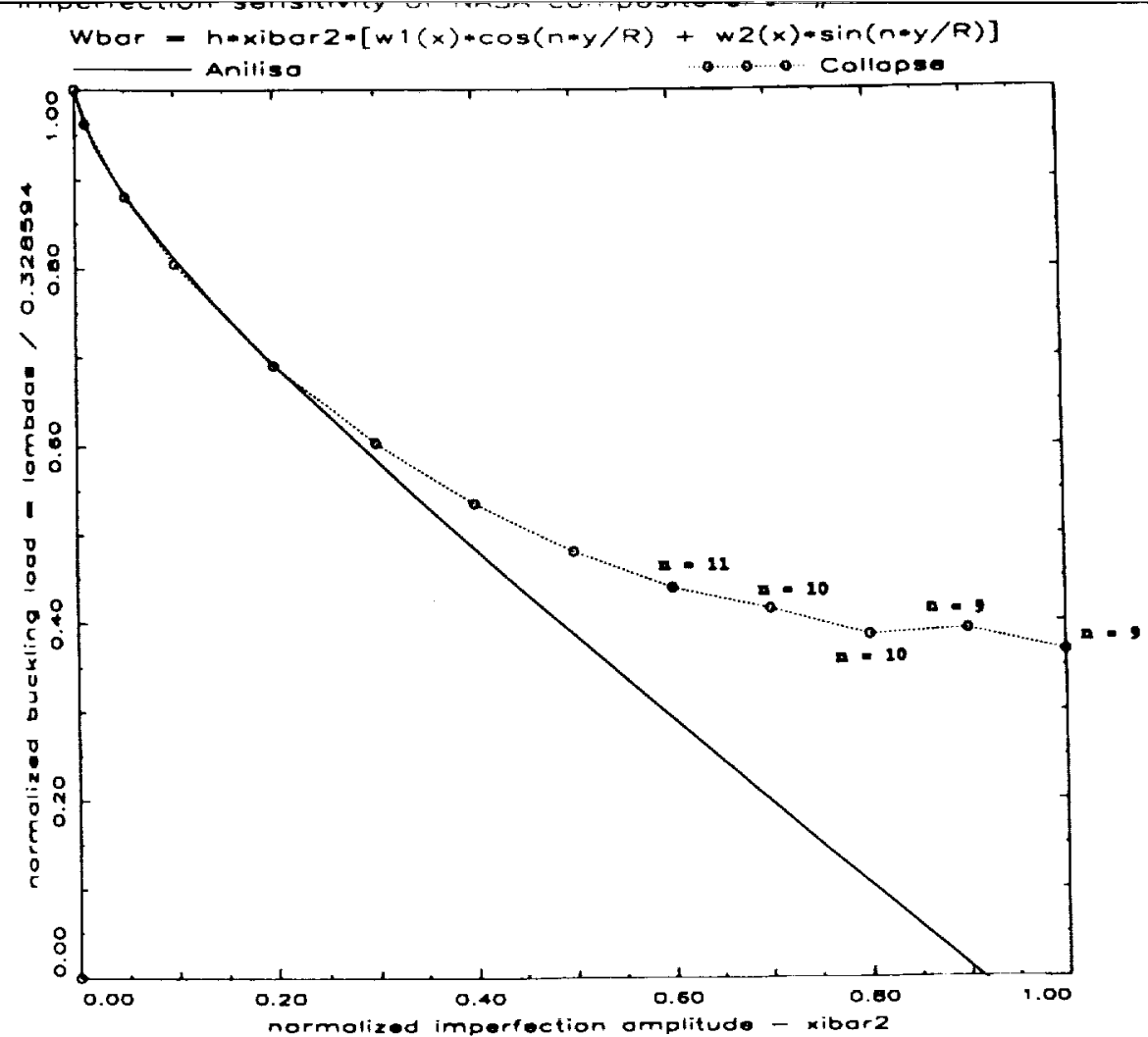

Fig. 9 Imperfection sensitivity calculation for affine asymmetric imperfections Asymptotic theory vs Nonlinear Collapse Analysis

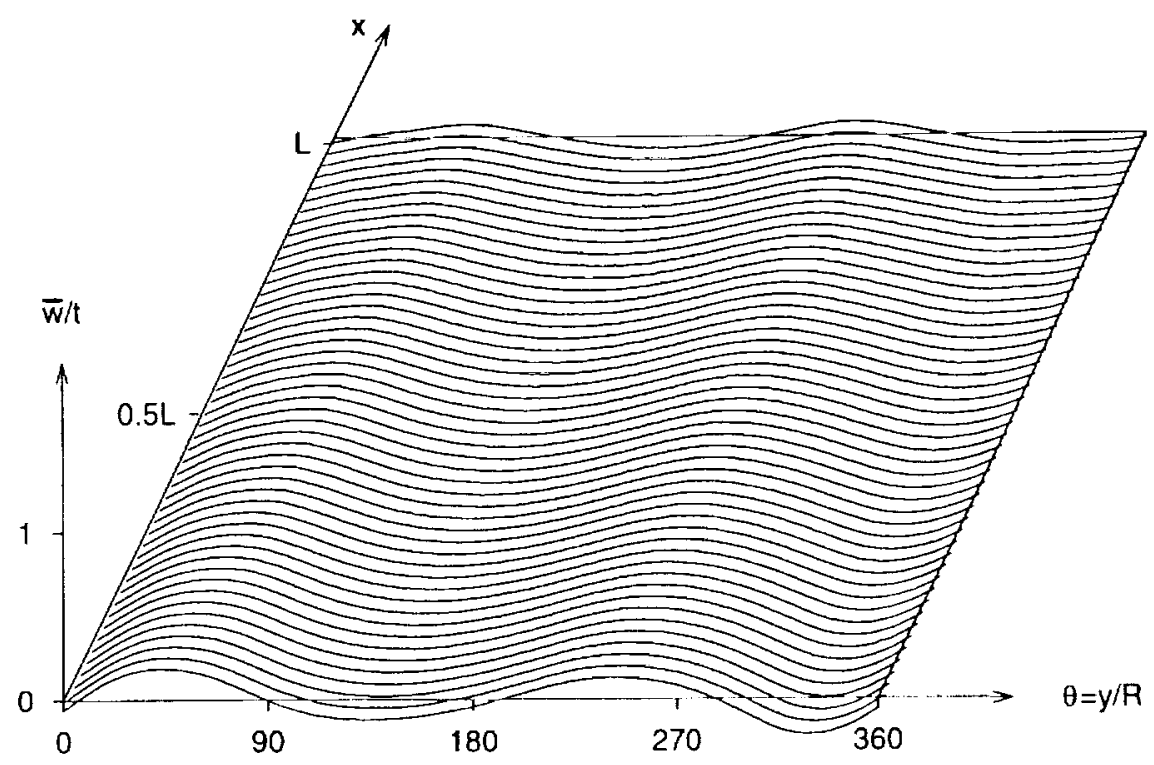

Fig. 10 Measured initial imperfections of NASA composite shell AW-CYL-1-1 [1] 


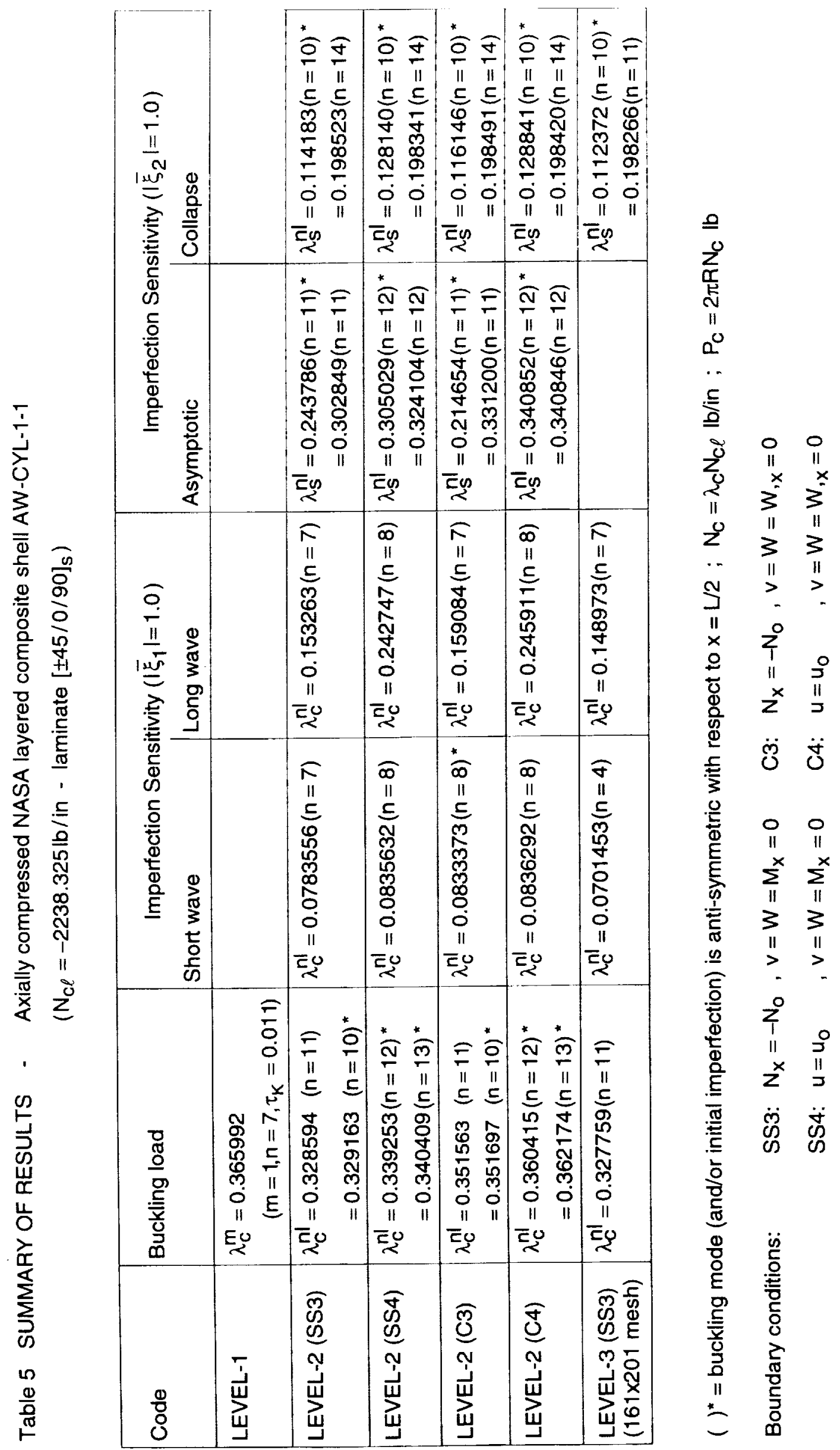




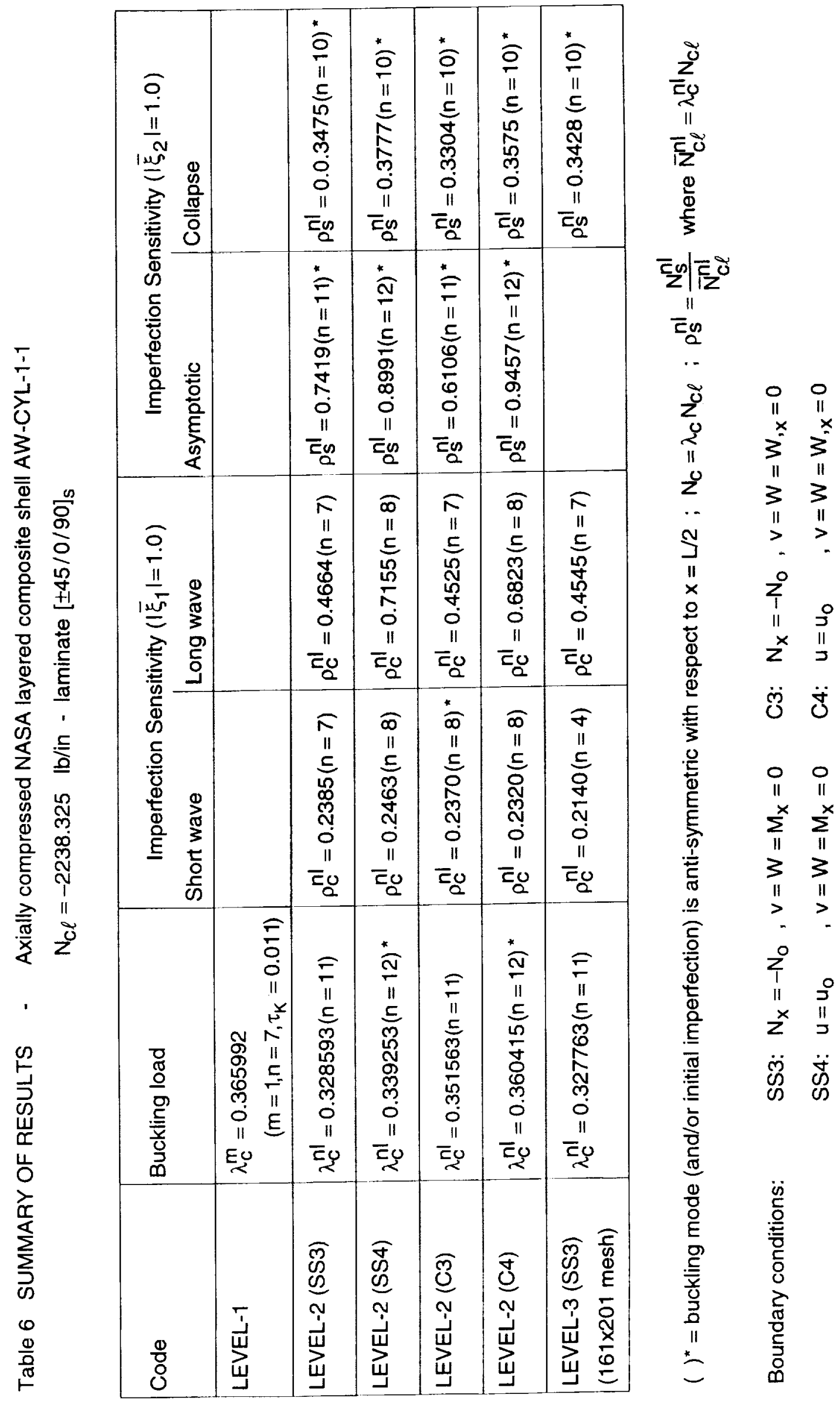

